\title{
MODEL OF ARTIFICIAL INTELLIGENCE MEDIUM AND ITS USE IN TREATING DISORDERS OF THE NERVOUS SYSTEM
}

\author{
Vladimir Shumilov ${ }^{\mathrm{a}}$ \\ National Research Tomsk State University, 634050, Tomsk, Russia
}

\begin{abstract}
The work is devoted to modeling of the nervous system, the brain. The article considers the mechanism of formation of event traces fixed in the brain in the form of connections between neurons and the effect of these traces on the later passage of signals through the brain. That is, the influence of experience (traces) of previous events on the organism's reaction by forecasting upcoming events. This «forecast» and anticipatory avoidance of dangers based on traces of previous events makes the nervous system, the brain useful for the organism, for its survival and expansion. The author proposes to use a computational model of the brain to study disorders of the nervous system, brain, and make recommendations for their prevention.
\end{abstract}

\section{Introduction}

Currently, the number of patients suffering from different diseases of the nervous system increases for a variety of reasons. Therefore, study of diseases of the human nervous system becomes more urgent [1-4]. However, scientists study the problem mainly by means of statistical methods, as well as post-mortem examination of brain pathologies, blood vessels and other organs of the deceased person. Now, there is a great number of different models of nervous systems. Scientists have made careful studies of diverse aspects of the structure and function of nervous systems of various biological organisms. Biochemical processes that provide function of the nervous system are of special attention. Researches investigate behavior of neurons - the main operating units of the nervous system, formation of connections between them, functions of various accessory tissues found in the brain. Neurogenesis - formation of new neurons in the human brain and in the brain of mature animals has been under active study lately, though until recently there has been an opinion that nerve cells of adults do not form, but only die. There is a great number of printed works devoted to various aspects of the processes occurring in the human body. Scientists have achieved great progress in research of various processes in the organism, in the brain. However, the logic of function of the nervous system is lost in numerous studied details and remains unclear. In our opinion, lack of understanding of mechanisms of the nervous system function in general and the brain in particular, is a big obstacle for treating diseases of the nervous system.

\footnotetext{
${ }^{\text {a }}$ Corresponding author: vnshumilov@rambler.ru
} 
We propose modeling method for study of diseases of the nervous system, which may significantly influence prevention, medical treatment, or at least retardation of development of these diseases. We see this way in use of the computational and electronic models of the nervous system. Within this approach, we represent the brain in the form of a huge electric circuit composed of active units - neural-like units and connections between them.

We have previously proposed principles of function of biological nervous systems and, in particular, the human brain [5,6]. These principles allow us to provide theoretical explanations of all or almost all aspects of operation of the biological nervous system and the brain. We should note that other researchers have also considered the principles and models of the nervous system, similar to those proposed by us ([7-9]). However, they have not suggested any mechanisms of self-formation of connections between neurons, mechanisms of forgetting and retraining. Therefore, they have not implemented their models either as any kind of software or as an electronic device.

Many processes in biological organisms provide function of the brain: mechanical (body movements and mass transfer by blood and lymph), biochemical and electrical. This happens because resources of the organism are limited, so it has to be economical - the same mechanisms are simultaneously involved in various processes. Each subsystem of the body has to perform a variety of functions through interactions with other subsystems in conditions of tight requirement of minimization of resources usage. Thus, blood supplies organs with nutrient, takes away organs' metabolic waste, heats and takes away the heat, protects the organism from foreign bodies, and moves various stimulants within the organism. The organism has to stand cold and hunger, overeating and overheating, poisoning and dehydration, physical overload and microorganisms' attacks in conditions of tight restriction on the organism's resources. Hence, the process of resources saving leads to combing of functions and incredible complication. It would seem that in order to construct a model of the brain it is necessary to consider all various processes running in the brain.

In fact, dealing with any problem, you can ignore many factors and not consider them for the reason that they have no or little influence on the parameters studied in the task. If, however, you cannot ignore the influence of some factors, you can consider the interaction of these factors by means of introduction of processes of equivalent substitutions, which replace the transitional conversion of considered parameters. The method of replacing substitutions is used to solve various problems. It can be used as well for considering information processes in the brain, in the nervous system. Almost any modeling is based on the use of substitutions.

\section{Characteristics of the brain}

Let us consider our proposed model of the processes occurring in the nervous system and in the brain. For more than 60 years, scientists have undertaken efforts to create artificial intelligence (AI). However, despite the continuing efforts of the specialists and success in particular cases, AI of full value has not yet been created. Despite the low performance of brain's active elements - neurons (the response time of a single neuron is about $0.1 \mathrm{sec}$.), parallelism of brain structures allows the brain to solve the problem of recognition, to respond to signals from the environment, to manage rapid body movements (running, jumping) for seconds, or even fractions of a second.

Principles of AI's algorithmic and hardware implementation are radically different from the principles of function of the biological brain. Therefore, even if within the current mainstream the AI is created, its study will have low effect for understanding function of the brain, areas of which obviously operate in parallel. Both human and animal brain does not require a big team of highly qualified specialists to provide its activity, but 
implementation of AI requires it. Moreover, efforts to build AI by traditional ways seem uneconomic to us. Indeed, the possible volume of information in the brain is large (at least 300 terabytes [5]), so processing of information contained in the human brain and its placement in the device with $\mathrm{AI}$, will require thousands of man- years of work of highly qualified specialists.

Therefore, we offer a completely different approach to building a model of the nervous system, based on understanding of mechanisms of function of the nervous system and brain. However, it is necessary to understand the principles of operation of these mechanisms. It is necessary to understand how the nervous system has appeared by a natural way without intervention of supernatural forces in full compliance with the objective laws of nature, and how it has passed the distance from the elementary nervous system to the brain of highly developed animals and humans. In addition, it is necessary to understand how the intellect appears in the brain formed naturally during millions of years of evolution.

Today, the continuing active search of ways for AI creation shows, that there is no common view on the logic of function of the nervous system (NS), on the logic of interaction of neurons (the simplest organisms), and even on the logic of function of the human brain. So far, the brain or the NS looks like a black box, despite the fact that the anatomical structure and biophysical processes in the NS and in the brain are well known $[7,8]$. Therefore, we will try to find the principles of function of the brain by means of considering of the pre-history of occurrence of the nervous system.

Electrical signals generated by the active elements - neurons when they are excited, are spread in the brain. Each neuron has a great number of inputs - up to 10000 . Neuron is excited when the sum of potentials on its all inputs, that is, the signal on its total input, which acts on the neuron' nucleus, exceeds a threshold level. By means of spending of accumulated nutrients, an excited neuron makes a signal of certain energy (standard capacity and duration), which allows signals to propagate through the branching structures of the brain without rapid attenuation and without increase of the number of signals propagating through the structure of the brain. Too rapid attenuation will not allow signals to reach the addressee - the motor neurons at the output of the NS. Increasing number of signals as they move (with the average rate of reproduction signals greater than one) would cause self-excitation of the brain. Besides other facts, this means that the signals do not occur in the brain, they are only transmitted (transferred, transformed) by brain structures, but are not generated, initiated. The nerve impulses appear only at the NS input under the influence on input brain circuits - receptors of environmental factors (stimuli). Brain neurons can be excited also by direct influence of electric potential.

Muscles or endocrine glands activated by the NS or the brain determine the final reaction of the organism to the received input signals (stimuli). This fact means that the reaction of the organism's NS to a stimulus depends on the path of stimulus signal spread from the input to output of the NS. Therefore, the reaction of the organism depends on the configuration of input signals and structure of the NS (possible signals paths) at the time of signal passing through the NS. On the other hand, it is known that the brain structure changes over time. The number of connections between neurons increases especially rapidly during intensive training $[10,11]$. This means that new signal paths are formed in the NS (in the brain) during the training.

So, unlike existing today control units (where information is recorded to a special memory block, read and interpreted by another special block), mechanisms of fixation, storage and interpretation of information (signal propagation) in the biological NS are combined functionally and are not localized in any certain isolated areas of the brain; they are distributed throughout the volume of the brain! Information content of the brain is determined by the structure of the brain, that is, by connections between neurons - the active elements of the NS (between which neurons does communication exist and what is 
their weight). The number of connections in the brain areas responsible for a certain type of activity increases rapidly during training, acquisition of experience in this type of activity.

What is the mechanism of formation of connections between neurons conducting signals in the NS, in the brain? What is the principle of formation of connections - memory elements? What are the mechanisms of propagation of signals received by the brain, by its structures? Why and how does propagation of signals through the brain structures provide an optimal behavior of the organism adequate to circumstances? Obviously, this mechanism must be extremely simple, provided by a natural process. Simply, there is nowhere to take complex mechanism from. This can be particularly well illustrated by the example of the emerging body, after a few cycles of self-copying, when only a few neurons have appeared in the organism, and there is still no connection between them.

We think that productive approach is based on consideration of the evolution from the simplest organisms up to human beings (and forward?).

\section{Functioning logic elements of the brain}

As answers to these questions, in [5] we have proposed provisions about principles of function of the brain (NS), which differ from the traditional ones. In accordance with these provisions and the patent based on them [6], the team of scientists of Tomsk State University (TSU) has made research «Development of electronic circuits for creation of a working model of artificial neural-like element». During this work, the specialists have developed the electrical circuits of the model, manufactured and tested the electronic model of an elementary nervous system, consisting of three active neural-like elements (NE), paired connections between them - both existing and potential connections, which do not yet exist in its original state, but which can be formed under appropriate circumstances. The model implemented in the form of an electronic device, consisting of even a small amount of NE, has confirmed the validity of the proposed principles. It has confirmed the possibility of self-creation of connections - event traces (we can say - training and retraining), formation of new stronger connections under the change of circumstances.

The essence of principles of function of the elementary NS (stated in [5]) and the NS's usefulness for the organism can be displayed in several diagrams illustrating the formation of the event traces in the NS, and the impact of these traces on the formation of the NS's reaction to the events that occur later. Electronic model of the elementary biological nervous system based on artificial NE, which was created as a result of research in TSU, has the same operation principles.

Let us consider the diagram of states of two neurons («excited»/ «not excited») when exposed by initially existing input factors (similar to unconditioned reflexes), which are important for the organism controlled by these neurons. The diagram depicts time $t$ (Figure 1). 


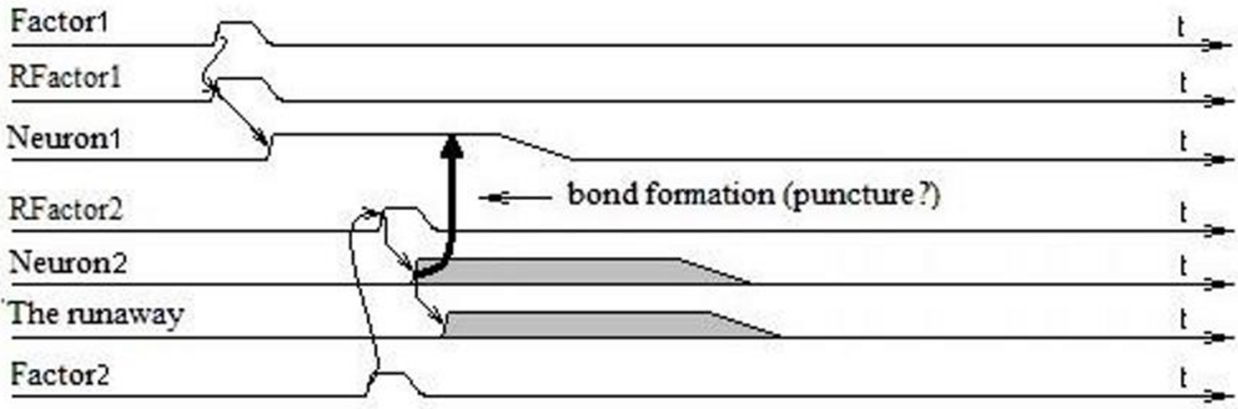

Figure 1. Excitation of neurons 1, 2 as a result of irritation of factors 1, 2 and formation of connection between the excited neurons.

Let Factor 1 precedes Factor 2, and Factor 2 is more dangerous for the organism than Factor 1. When Factors (stimuli) appear, they excite relevant neurons through separate inputs. Besides, appearance of Factor 2 causes unconditional response - the body moves away from Factor 2 so that Factor 2 will stop to influence the organism (its receptors). Neurons may have many inputs, through which can be excited.

What will happen with the organism later, if in the described episode the organism was able to avoid the lethal effects of Factor 2? What will happen if in this episode the event trace was formed, which means approximately simultaneous excitation of neurons $\mathrm{N} 1$ and $\mathrm{N} 2$ ? This trace is a logical connection between neurons 1 and 2, which is formed at a moment of simultaneous excitation of neuron. This connection is realized in real organisms biologically. In principle, in various models it may be formed in any possible way, for example, in mechanical, pneumatic, hydraulic, chemical or galvanic way through potentially possible new inputs. Moreover, at least two options of such connection are possible:

a) output of Neuron 1 (N1) to input of Neuron 2 (N2);

b) output of Neuron 2 (N2) to input of Neuron 1 (N1).

The probabilities of these options are determined by time correlations of stimuli F1, F2 (the order in which they have followed), and the relative position of inputs and outputs of neural-like elements. Let us consider the situation when option a) was realized.

Then, the next time in a similar situation, the events will develop in the following way. In case of excitation of one of already interconnected neurons (say, only N1 by factor F1), immediately after excitation of $\mathrm{N} 1$ another neuron $\mathrm{N} 2$ will be excited through the event trace formed previously, through existing connection (from output of N1 to input of N2), although harmful factor F2 still does not influence it. And maybe, even will not influence it in this episode. So the diagram given above will be as follows (Figure 2):

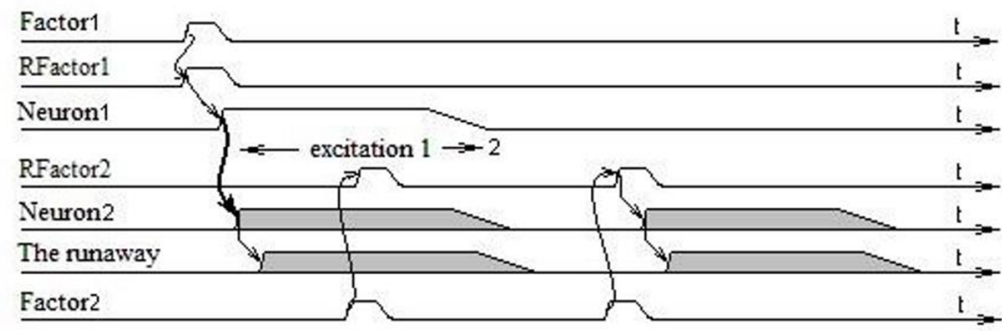

Figure 2. Excitation of Neuron 2 by Factor 1 through the previously self-formed connection N1 - N2. 
It means that the presence of the trace (previously formed connection between neurons) enables afterwards the following order of events. As a result of influence of Factor 1, N1 excited through the previously formed connection with N2, immediately excites it. The organism immediately responds to excitation of $\mathrm{N} 2$ and «escapes», moves away from Factor 2, although Factor 2 has not yet appeared, and may not appear. If it appears, it will not have a harmful effect on the organism due to «provident», but actually just leading «runaway» from the approaching harmful factor. So the organism with the elementary nervous system of two neurons respond to Factor 2 IN ADVANCE, AHEAD as a result of previously gained experience - formation of the event trace, formation of connection between two excited neurons $\mathrm{N} 1$ and N2 (which may be called elementary memory or memory element). As if the organism foresees appearance of F2 due to formation in the previous episode and further existence of communication N1-N2, which is equivalent to memorization of fact of simultaneous action of Factors 1 and 2 in some episode occurred earlier. This leading and, thus, anticipatory to danger (providing provident avoidance of danger) reaction of the organism with the elementary nervous system capable to fix the event traces (to «remember»), will undoubtedly contribute to a more successful survival of the organism and its further expansion in spite of the possible emergence of dangerous Factor 2.

Organisms exist in the material world where events occur due to cause-and-effect relationship of phenomena, so not random, uncoordinated and unconnected events occur in nature, but the chains of causal events. That is, the event $\mathrm{A}$ is usually (depending on the surrounding circumstances) followed by the entire sequence of causal events B, C, D, etc. Therefore, the same events- effects usually follow the same events-reasons. For example, smell precedes burning; view and sound of a flying stone precede stroke of the same stone; a flash of lightning (signal speed of $300,000 \mathrm{~km} / \mathrm{sec}$ ) precedes the sound of thunder (speed of $0.33 \mathrm{~km} / \mathrm{sec}$ ). The fact that events are connected in recurring chains provides the body with the ability to detect the approach of a dangerous event and move away from it in advance by detecting some events (probably not dangerous) of this sequence, which precede danger. The leading detection of dangerous events by the organism (forecast) is possible if the previous passage of events chain has left a trace.

Formation of such trace, connection between two neurons is possible under the following conditions. In the natural environment in usual visible macroscopic life, everywhere we can see traces, which have significant effect on the course of events. For example, water flows along the existing paths - furrows, gullies, channels of streams and rivers; rut on the road affects cars movement.

Usually, or at least sometimes, Factor 2 from our example will follow Factor 1 because of the causal event conditionality. However, the organism in advance, even before the arrival of harmful Factor 2, will «predict» appearance of Factor 2 after appearance of Factor 1 by the trace left in the organism during the previous episode. So the organism will provide «foreseeing» forward-reaction to Factor 2 (sometimes in vain, because the harmful Factor 2 may not occur) and «avoid», move away from it, saving its life, continuing later in progeny, and repeating in progeny very useful ability to fix traces of events - to remember pairs of simultaneously operating factors.

This is the useful function of the nervous system of organisms with memory "foreseeing» (anticipatory reaction) based on previous experience.

It should be noted that we solve almost all challenges facing us upon the model or combination of models (their traces), which we have met earlier as a result of our experience, during the course of explicit or implicit learning. 
In order to fix the trace of a random event by forming connections between neurons excited by these events, the inputs and outputs of biological neurons or NE in electronic circuits must form a lattice of possible connections, as shown on Figure 3:

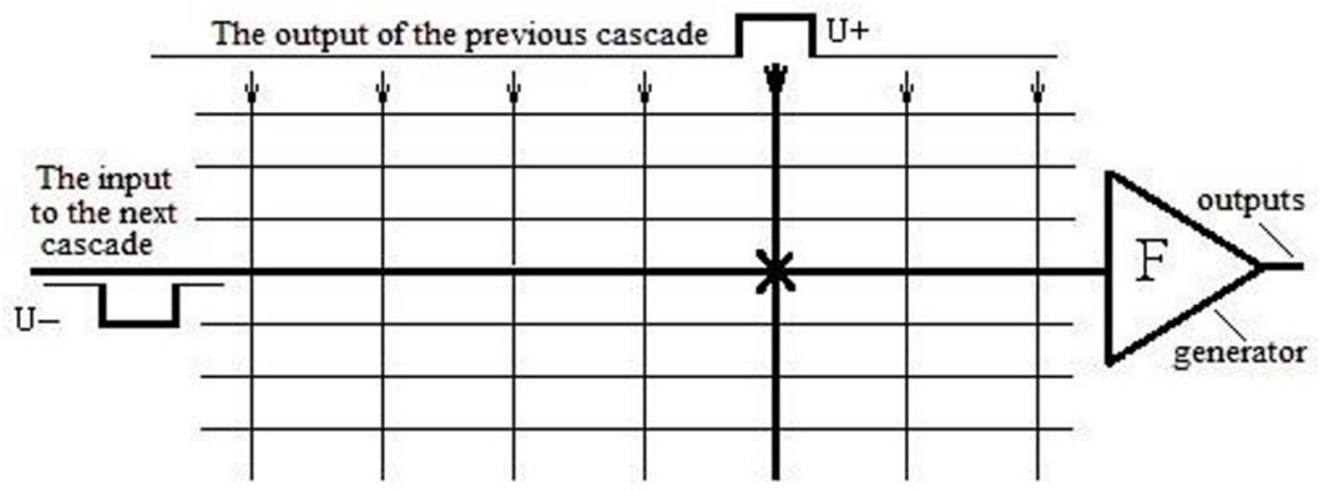

Figure 3. Formation of connections between two random excited NE, outputs and inputs of which are isolated in the initial state.

How can relationship, an event trace, which means simultaneous excitation of two NE, be formed? Figure 4 shows one of the options of fixation, formation of such event. This option of formation of connection between the excited elements has been implemented in the electronic model of the elementary nervous system, created as a result of R \& D. This mechanism ensures formation of connection between two simultaneously excited NE.

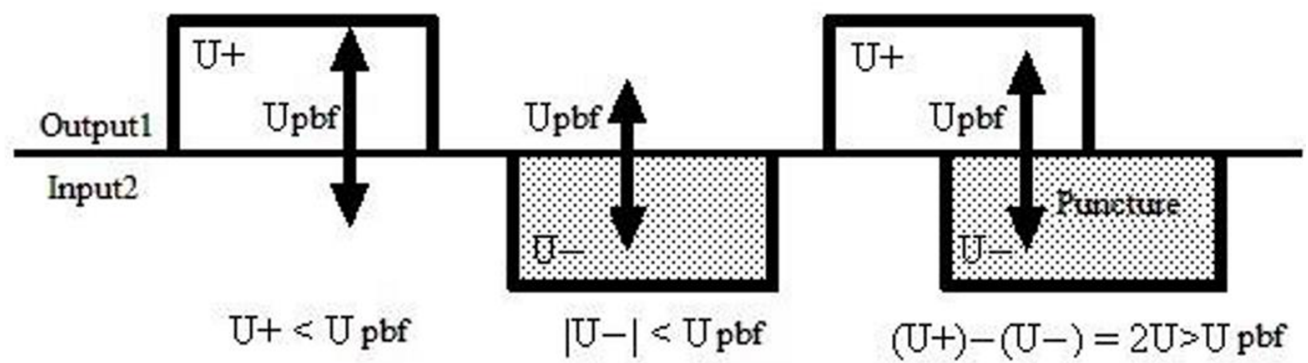

Figure 4. Formation of connection between two simultaneously excited neurons.

Figure 4 illustrates situation when the difference of potentials between two points (Output 1 and 1 Input 2 equal to $(\mathrm{U}+)-(\mathrm{U}-))$, at a certain period of time exceeds a certain value $U_{p c f}$ (potential of connection formation). Absolute values of potentials of these two points separately are less than $U_{p c \text { f. }}$ So breakdown or another irreversible phenomenon realizing formation of connection - «remembering» (fixation) of an event, can take place only for two simultaneously excited NE, the difference of potentials at inputs and outputs of which exceeds $U_{p c}$, and which have mechanical contact (these make formation of connection possible). This simple mechanism provides self-connection between two excited NE, most likely between two biological neurons. Even if a new connection is formed not by means of the breakdown, logical connection can be formed only by means of the described method. 
It should be noted that formation of connection with real load characteristics results in the fact that potentials of inputs and outputs of NE cannot remain unchanged throughout the whole period of NE excitation, and they are no more regular rectangular. This is because of the mutual compensation of potentials on output and inputs of excited neurons. Figure 5 shows this fact.

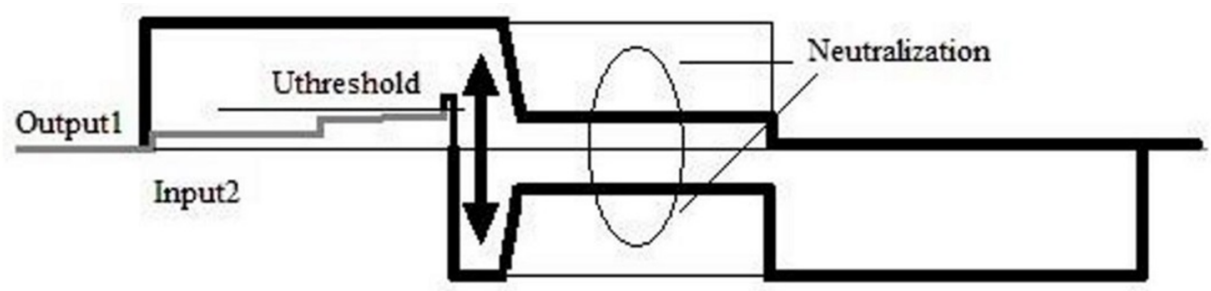

Figure 5. Neutralization of potentials of inputs and outputs of excited NE during formation of connection.

Mutual compensation of potentials on outputs and inputs of two excited NE, shown on Figure 5, provides an opportunity to solve many related problems, both in NS of living organisms, and the NS model implemented based on electronic NE. This model of the elementary NS is based on three electronic neural-like elements, the effect of which is completely transparent and understandable. This simple model shows the possibility of «learning» and «retraining» of the elementary NS implemented as an electrical circuit and acting in accordance with the above-described principles.

As the created electronic model has demonstrated efficiency of the proposed principles of the brain function, it is possible to represent the brain in a form of a very large-scale electric circuit. Figure 6 shows this circuit. 


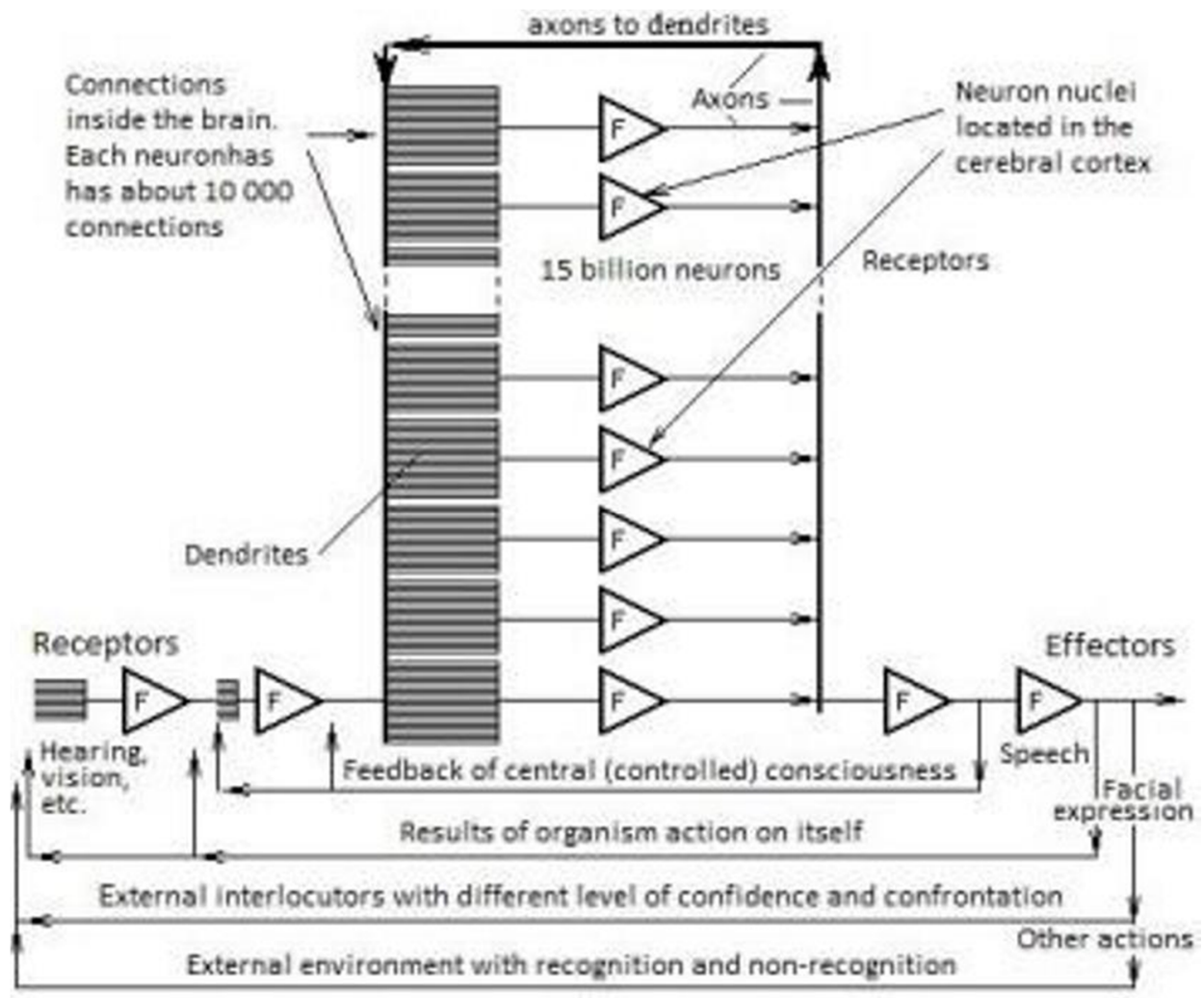

Figure 6. Electric diagram of the human brain.

\section{Intellection}

Neurons with only local mechanisms cannot determine where signals come to input from. Therefore, all neurons together with their connections act in the same way, no matter where exciting signals come from. Function of each neuron is determined by configuration of connections on its inputs and by configuration of signals coming on these inputs. Neural connections are traces of previous experience of the organism. All neurons being excited, «make their forecast» based on configuration of the current signals (events) and previous experience. Every time these forecasts are single-staged, single-linked. But excitation signals of any neurons in a large electronic circuit of the brain can and act as excitation factors for subsequent neurons, and thus, they create the following links of single-staged forecasts. These single-staged forecasts are combined into long, sometimes intersecting, multi-staged forecasts. Signals passing through such long chains represents thoughts, fragments of thoughts.

With a large number of elements in this circuit, «a thought» being generated by an external factor can move along the circuit for a long time and form new connections between active elements. Configuration of input signals and structure of connections between active elements (neurons), established at the time of passage, that is, the individual «experience» of the scheme define paths of signal passage along the circuit. So the signal originally generated by hunger or splinter when crossing with other chains can turn into a thought about advanced training, a needle, metals, and so on to the structure of the universe through planes and space ships. 
Taken into account the huge amount of connections (about $1.5^{*} 10^{14}$ ) and the need to address them (at least 2 bytes per indication of source signal for each possible connectiondendrite in the human brain), it becomes obvious that creation of a device that simulates function of the human brain by direct installation of all these connections is almost impracticable. With so many possible connections it turns out that even with the period of brain life of about 100 years, the average rate of formation of connections between neurons is about 30,000 connections per a second. These connections are formed in the brain as a result of acquisition of experience - training, upbringing, activity in general. Therefore, we propose another way. To simulate function of the brain we should create a device with the same potential for development as the natural human brain. But, as well as the brain, the model in the initial state is not ready for full operation. However, it is ready for development. During the process of the model creation, we set connections, which realize likeness of unconditioned reflexes of newly born babies. Next, based on connections, which realize unconditioned reflexes, we bring up reaction-skills in this device necessary for its comfortable existence in the environment, just as people and animals raise their babies. That is, to bring up intelligence in the device, not to create the ready intelligence.

In order to strengthen conclusiveness of correspondence of the created models to real biological nervous system, we should confirm in detail correspondence of the models to biological nervous systems in existing living organisms. For confirmation, we need to obtain neuron input/output potential graphs of the simplest biological organisms, such as California sea hare (Aplysiidae). It is necessary to do so in order to ensure that potential graphs of biological nervous systems and of our models correspond to each other. We would like to obtain potential graphs when there is excitation of neurons, especially at the moment of formation of new connections between neurons.

Currently, we are carrying out this work.

Confirmed correspondence of software and electronic models of the biological nervous system will allow more confident studying of the behavior of biological nervous systems on the computational model.

If we are confident that our models reflect processes of biological nervous systems perfectly true, then it will be possible to make recommendations on diagnosing and treatment for biological nervous systems diseases based on the study of models' behavior. Even now, we can give some recommendations concerning ways of medical treatment for diseases. If a person's mechanism of new events memorization has failed but the person remembers rather well events passed a long time ago, we can make the following conclusion. According to our model of the nervous system function - formation of new connections between neurons as memory elements - the memory disorder may be caused by the fact that formation of new connections in the nervous system in the long-term memory area of the brain has stopped for some reason.

The reason could be in reduction of value of excited neurons potential (for example, because of increase of leakage). The value of these potentials becomes insufficient to form new connections in this area [12]. On the other hand, inability to form new connections may be caused by increase of the value of the potential of a new connection formation. For example, by increase of breakdown voltage of membranes separating neighboring neurons.

In both cases, there is need for a certain change of biochemical conditions in the brain, which could change the existing conditions of the brain and restore the ability of brain structures to form connections.

\section{Conclusion}

The problem of study of diseases and ways of medical treatment is the responsibility of medical researchers. Our task is to provide medical researchers with a convenient tool for 
studying of various diseases of the nervous system on the computational model, allowing quick change of various parameters that affect the memory model function, and observation the model's reaction on change of these parameters. Currently, we are also carrying out work on creation of the software model of the nervous system with convenient for medical researchers interface.

Together with biologists' work on confirmation of correspondence of processes of biological nervous systems function with our models, it will create a good platform for a humane (non-traumatic intervention in the life of a biological organism), economical, effective (efficient) study of various diseases and disorders of the nervous system, their prevention and medical treatment.

\section{Acknowledgements}

The paper was written as part of the research project No. 8.2.31.2015, carried out with the support of the Program "Research Foundation of Tomsk State University named after D.I. Mendeleev" in 2015 - 2016.

\section{References}

[1] L. A. Amaducci, W. A. Rocca and B. S. Schoenberg, Neurology 36, 1497 (1986)

[2] B. E. Tomlinson, G. Blessed and M. Roth, J. Neurological S. 11, 205 (1970) doi: 10.1016/0022-510X(70)90063-8

[3] F. Matthews and C. Brayne, PLoS Med 2, 0753 (2005) doi: 10.1371/journal.pmed.0020193

[4] Dementia UK (Alzheimer's Society, London, 2007) URL: http://www.alzheimers.org.uk/site/scripts/download_info.php?fileID=2

[5] V.N. Shumilov, Printsipy funktsionirovanija mozga. Vzgljad inzhenera (The principles of functioning of the brain. Sight of engineer) (Ekaterinburg, 2008)

[6] V.N. Shumilov, V.N. Voronin, V.I. Solomonov, The adaptive controller, the base neuro-similar element and a method of organizing the work of such a device RF Patent № 2475843

[7] A. V. Zhdanov, Science and Life (1), 2000 URL: http://www.nkj.ru/archive/articles/5613/

[8] M. P. Van Den Heuvel, C. J. Stam, R. S. Kahn and H. E. Hulshoff Pol, J. Neuroscience 29, 7619 (2009) doi: 10.1523/JNEUROSCI.1443-09.2009

[9] Jeff Hawkins, Sandra Blakeslee, On Intelligence (Macmillan, New York,2007)

[10] The growth of axons in the developing cerebral cortex of mammals URL: http://scorcher.ru/axiomatics/axiom_show.php?id=305 (15.03.2015)

[11] Psychophysiology, University textbook, edited by Y. I. Alexandrov (Piter, SPb,2010)

[12] V. N. Shumilov; V. I. Syryamkin, M. V. Syryamkin, New operational technologies (NEWOT'2015) 1688, 040007 (2015) doi: 10.1063/1.4936040 